\title{
BMJ Open Alcohol and the risk of pneumonia: a systematic review and meta-analysis
}

\author{
Evangelia Simou, John Britton, Jo Leonardi-Bee
}

\begin{abstract}
To cite: Simou E, Britton J, Leonardi-Bee J. Alcohol and the risk of pneumonia: a systematic review and meta-analysis. BMJ Open 2018;8:e022344. doi:10.1136/ bmjopen-2018-022344

- Prepublication history and additional material for this paper are available online. To view these files, please visit the journal online (http://dx.doi. org/10.1136/bmjopen-2017022344).
\end{abstract}

Received 14 February 2018 Revised 29 May 2018 Accepted 18 June 2018
D) Check for updates

(c) Author(s) (or their employer(s)) 2018. Re-use permitted under CC BY. Published by BMJ.

Division of Epidemiology and Public Health, UK Centre for Tobacco and Alcohol Studies, University of Nottingham, Nottingham, UK

\section{Correspondence to} Miss Evangelia Simou; evangelia.simou@nottingham. ac.uk

\begin{abstract}
Objective A systematic review and meta-analysis to estimate the magnitude of the association between alcohol consumption and the risk of community-acquired pneumonia (CAP) in adults was undertaken.

Design Systematic review and meta-analysis.

Methods Comprehensive searches of Medline,

Embase and Web of Science were carried out to identify comparative studies of the association between alcohol intake and CAP between 1985 and 2017. Reference lists were also screened. A random-effects meta-analysis was used to estimate pooled effect sizes. A dose-response meta-analysis was also performed.
\end{abstract}

Results We found 17 papers eligible for inclusion in the review, of which 14 provided results which could be pooled. Meta-analysis of these 14 studies identified an $83 \%$ increased risk of CAP among people who consumed alcohol or in higher amounts, relative to those who consumed no or lower amounts of alcohol, respectively (relative risk $=1.83,95 \% \mathrm{Cl} 1.30$ to 2.57 ). There was substantial between-study heterogeneity, which was attributable in part to differences in study continent, adjustment for confounders and pneumonia diagnosis (clinical vs death). Dose-response analysis found that for every 10-20 g higher alcohol intake per day, there was an $8 \%$ increase in the risk of CAP.

Conclusions The findings suggest that alcohol consumption increases the risk of CAP. Therefore, strengthening policies to reduce alcohol intake would be likely to reduce the incidence of CAP.

\section{INTRODUCTION}

Pneumonia is a major cause of global morbidity and mortality. In 2014 in the USA, pneumonia (including influenza) was the eighth leading cause of death, ${ }^{1}$ and according to the WHO, in 2015 pneumonia was responsible for $16 \%$ of all deaths in children aged under 5 years. ${ }^{2}$ Community-acquired infections are the most common cause of pneumonia, and with an annual incidence in Europe and North America of between 5 and 11 cases per thousand adults, ${ }^{3}$ community-acquired pneumonias (CAPs) account for a total of 4 million deaths annually. ${ }^{4}$ Globally, Streptococcus pneumoniae is the most common pathogen causing $\mathrm{CAP}^{5}{ }^{5}$ The annual incidence of CAP requiring hospitalisation among US adults is 24.8 cases per 10000

\section{Strengths and limitations of this study}

- This study represents a comprehensive review of the global literature with no language restrictions, while adhering to the guidelines of the Preferred Reporting Items for Systematic Reviews and MetaAnalyses and the Meta-analysis Of Observational Studies in Epidemiology.

- Heterogeneity was explored using subgroup analysis based on a priori defined factors.

- A dose-response analysis of alcohol consumption was also performed.

- Confounding as a result of the existence of other factors that were not usually adjusted for in the included studies (eg, socioeconomic status, malnutrition) could not be explored.

adults, with the highest incidence especially in oldest people. ${ }^{6}$ Patients with severe CAP admitted to European intensive care units have a mortality rate of $27 \%$ at 6 months. ${ }^{7}$

Pneumonia is more common with increasing age, ${ }^{8} 9$ among people who smoke, ${ }^{10-12}$ have low body mass index, ${ }^{13}$ or have comorbidities including other respiratory diseases, ${ }^{12}{ }^{14}$ cardiovascular diseases, ${ }^{14}$ stroke, ${ }^{14}$ dementia, ${ }^{11}{ }^{14}$ and liver or renal disease. ${ }^{14}$ Alcohol consumption is a potential risk factor for pneumonia. There are several possible mechanisms to explain the observation that alcohol consumption increases the risk of pneumonia, including the sedative properties of alcohol which can reduce oropharyngeal tone, leading to an increased risk of aspiration of microbes. Furthermore, high levels of alcohol intake can modify alveolar macrophage function, hence diminishing pulmonary defence against infection. ${ }^{15} 16$ Also, high alcohol consumption is often associated with malnutrition ${ }^{17}$ as it interacts with nutrient metabolism and utilisation, ${ }^{18}$ resulting in the impairment of immunity and increased CAP risk.

To date, however, evidence on the association between alcohol consumption and CAP is limited. A systematic review and meta-analysis published in 2010, using evidence published before August 2009, found a 6\% increase in 
the risk of pneumonia per standard drink of $12 \mathrm{~g}$ of pure alcohol per day, but the number of studies reviewed (five) was small. ${ }^{19}$ However, there is an increase in the interest on this topic, and also several studies have been published in the past 9 years. For this reason we have carried out a systematic review and meta-analysis to quantify the association between alcohol consumption and the risk of CAP.

\section{METHODS}

The systematic review and meta-analysis was carried out in adherence to the guidelines of the Preferred Reporting Items for Systematic Reviews and Meta-Analyses ${ }^{20}$ and the Meta-analysis Of Observational Studies in Epidemiology ${ }^{21}$. The protocol was published in the National Institute for Health Research international prospective register of systematic reviews (PROSPERO) under PROSPERO registration number 42015029910.

\section{Patient and public involvement}

No patients or the public were involved in this review.

\section{Inclusion criteria}

The Population-Exposure-Outcome-Study Design (PICO) criteria were used to determine eligibility of the articles based on the type of study design, type of population, type of exposure and outcome. We included all comparative study designs (longitudinal, cohort, case-control and cross sectional) assessing the association between alcohol intake and the risk of CAP in generally representative adult populations ( $\geq 18$ years), and therefore excluded studies of selected populations such as people with HIV, hepatitis $\mathrm{B}$ or $\mathrm{C}$ virus infection, and those with hospital-acquired pneumonia. Where possible, we also analysed the association between alcohol consumption and the occurrence of pneumonia due to specific organisms (eg, S. pneumoniae) .

\section{Exposure ascertainment}

Alcohol consumption was defined either by self-report (interview or questionnaire) or using medical records. Also, alcohol use corresponded to drinking levels (low, moderate, heavy and alcoholism) or to frequency measures (grams/units/drinks per day/week).

\section{Outcome ascertainment}

CAP diagnosis was based on clinical diagnosis (chest X-ray, blood test), physician diagnosis and medical records including the International Classification of Diseases (ICD) codes, or self-report.

\section{Search strategy}

Comprehensive search strategies were applied to Medline (via Ovid), Embase (via Ovid) and Web of Science databases for the period from December 1985 to December 2017. We used search filters for observational study designs ${ }^{22}$ and search terms for both outcome and exposure developed from relevant Cochrane Review groups. ${ }^{23}$ When searching, medical subject heading terms were used for Medline and Embase, whereas freetext words were used for Web of Science. The Medline search filters were the following: [exp Alcohol-Related Disorders/OR Alcohol Drinking/OR (alcohol adj3 (drink\$ ORor intoxicat\$ OR use\$ OR abus\$ OR misus\$ OR risk\$ OR consum\$ OR withdraw\$ OR detox\$ OR treat\$ OR therap\$ OR excess\$ OR reduc\$ OR cessation OR intervention\$)).tw. OR (drink\$ adj3 (excess OR heavy OR heavily OR harm OR harmful OR hazard\$ OR binge OR problem\$) ).tw. OR alcoholic\$.tw.] AND [exp Respiratory Tract Infections/OR (acute respiratory infection*.tw.) OR (lower respiratory infection*.tw.) OR (lower respiratory tract infection*.tw.) OR exp Pneumonia/OR (pneumon* OR bronchopneumon* OR pleuropneumon*).tw. OR exp Bronchitis/OR (bronchit* OR bronchiolit*).tw]. The full search strategy is presented in online supplementary table E1). The reference lists of included studies were also screened in order to identify further potentially eligible studies. No language limitation was imposed, and where necessary papers were translated into English. Where there was more than one report of findings from the same population (eg, an abstract and then a full paper), the most recently published version of the study was used. Screening of titles and abstracts, as well as the full text, was conducted independently by two reviewers (ES and JL-B). Any disagreements were resolved through discussion or with the help of a third reviewer (JB).

\section{Data extraction}

Two reviewers (ES and JL-B) independently extracted data using a previously piloted form (see online supplementary table E2), which included the following information: author, year, study design, definitions of exposure (alcohol) and outcome (CAP), geographical location, reference population, and adjustment for confounders.

For categorical measures of alcohol drinking, where possible we compared any alcohol consumption with no alcohol consumption (reference group), or else used the lowest exposed category as the reference group. Also, in the main analysis, categorical measures of alcohol consumption were further defined as levels of consumption: light, moderate, heavy, binge and alcoholism. Grams of daily alcohol consumption were used as a standard measure, defining one drink as 0.6 ounces, $14.0 \mathrm{~g}$ or 1.2 tablespoons of pure alcohol. ${ }^{24}$ Where possible, we followed the Centers for Disease Control and Prevention guidelines for the definition of heavy drinking as a weekly consumption of 15 or more drinks for men, and 8 or more drinks for women; binge drinking as 5 or more drinks during a single occasion for men, or 4 or more for women; and excessive drinking as the presence of either binge or heavy drinking. ${ }^{24}$ The Dietary Guidelines for Americans define moderate alcohol drinking as the daily consumption of up to one drink for women and two drinks for men. ${ }^{25}$ Otherwise we accepted the definitions of alcohol that the included studies used. 


\section{Quality assessment}

Two authors (ES and JL-B) independently assessed the methodological quality of the included studies using the Newcastle-Ottawa Scale. ${ }^{26}$ In the process of quality assessment of each article, a maximum score of 9 stars can be obtained, whereas studies with lower quality obtain fewer stars. In case of a cohort study, the cohort study criteria were used, whereas for case-control studies the case-control criteria were used. However, for a cross-sectional study, a modified version of the case-control study criteria was used and in this case a maximum of 7 stars was given. All studies, irrespective of their design, were considered to be of high quality if they obtained a score of $\geq 6$ stars. Discrepancies were resolved through discussion and consensus. We did not attempt to assess the methodological quality for studies published only in abstract form.

\section{Statistical analysis}

Relative measures of risk were extracted as ORs, relative risks (RR) or HRs with $95 \%$ CIs. Where available, we used measures of risk adjusted for smoking and socioeconomic status and extracted the results separately for men and women. Where raw data were extracted from studies, we estimated ORs for case-control studies and RRs for longitudinal, cohort and cross-sectional studies. Where exposure to alcohol was reported using quantiles, or categories, we extracted adjusted effect measures relating to a comparison of the highest with the lowest exposure group.

The pooled RR and the $95 \%$ CI were estimated through pooling ORs and RRs together, since it was assumed that these two measures of effect would be similar due to the outcome measure being uncommon (prevalence $<\sim 10 \%) .{ }^{27}$ However, HRs were not pooled with other effect measures. The decision to present only relative risks was made due to issues associated with using absolute risks, namely the risk difference is naturally constrained which may create difficulties when applying results to other patient groups and settings. Therefore, absolute measures are less likely to be generalisable. ${ }^{28}$ Meta-analysis was conducted, based on the DerSimonian and Laird's random-effects model, to pool the results from the individual studies.

Heterogeneity between studies was quantified using $\mathrm{I}^{2}$ statistics, ${ }^{29}$ and explored using subgroup analyses according to study quality, study design, adjustment for confounders, alcohol reference group (no alcohol vs lowest exposed category), CAP diagnosis (clinical diagnosis vs death records), geographical location (low-income and middle-income countries vs high-income countries) and measure of effect estimated (ORs vs RRs). Funnel plots were used as a visual aid to detect publication bias, and where data for at least 10 studies were available we formally assessed publication bias using Egger's asymmetry test. We performed all analyses using Stata V.14 and Review Manager V.5.3. All $p$ values $<0.05$ were deemed to represent statistical significance.

\section{Dose-response assessment}

To assess the evidence for causality, we applied a modified version of Hill's criteria to assess causation ${ }^{30}$ on strength of association, consistency, temporality, biological gradient and plausibility. To assess the biological gradient criterion, we performed a random-effects dose-response meta-analysis, ${ }^{31} 32$ where we assumed a linear dose-response relation and allowed for study-level correlations across the categories of quantities of alcohol. The dose-response relation between alcohol consumption and CAP was analysed using the subgroup of studies including at least three different categories of exposure, standardised for analysis to grams per day, and where appropriate using the midpoint of categories defined by ranges of intake. If the highest exposure category was open-ended, we took the highest category midpoint to be the lower bound plus 1.2 times the lower boundary. ${ }^{33}$ When available we included results for men and women separately.

Separate dose-response meta-analyses were conducted for cohort/longitudinal and case-control/cross-sectional studies. Dose categories relating to quantities of alcohol were created to equate to $10-20 \mathrm{~g}$ of pure alcohol per day (approximately one drink per day); where studies reported categories which contained the same dose ranges, we collapsed these into a single dose category through estimating a pooled effect estimates based on a fixed-effect meta-analysis model. Where necessary, effect estimates and $95 \%$ CI were back-calculated from floated to conventional CIs to enable comparisons to be made with the reference group (non-drinkers or the lowest exposed category). ${ }^{34}$

\section{RESULTS}

The searches identified a total of 4589 studies published between December 1985 and December 2017, of which 17 were eligible for inclusion in the systematic review (figure 1). The characteristics of the 17 included studies are presented in table 1. A total population of 287184 people were included in our review. Seven studies used a cohort or longitudinal design, ${ }^{10} 35-40$ nine used a case-control design ${ }^{1141-48}$ and one used a cross-sectional design. ${ }^{49}$ Eight studies were conducted in America, ${ }^{10} 11394046-49$ five in Europe, ${ }^{374143-45}$ two in Asia $^{3536}$ and two in Australia. ${ }^{384}$ Three studies reported separate estimates of the association between alcohol and CAP for men and women, ${ }^{10} 4144$ and 12 studies reported effect estimates adjusted for confounders. ${ }^{10} 3536394143-49$

The majority of studies assessed alcohol consumption by self-report, based either on a standardised questionnaire or on an interview, while five studies used reported intake data from medical records. ${ }^{1137404647}$ The reference group for nine studies comprised people who never consumed alcohol, ${ }^{103536384244454748}$ whereas the reference group for the remaining eight studies comprised people who consumed the lowest quantity of alcohol. ${ }^{11} 3739-41434649$

Seven studies ascertained CAP using a clinical diagnosis, and five of these used chest X-ray 


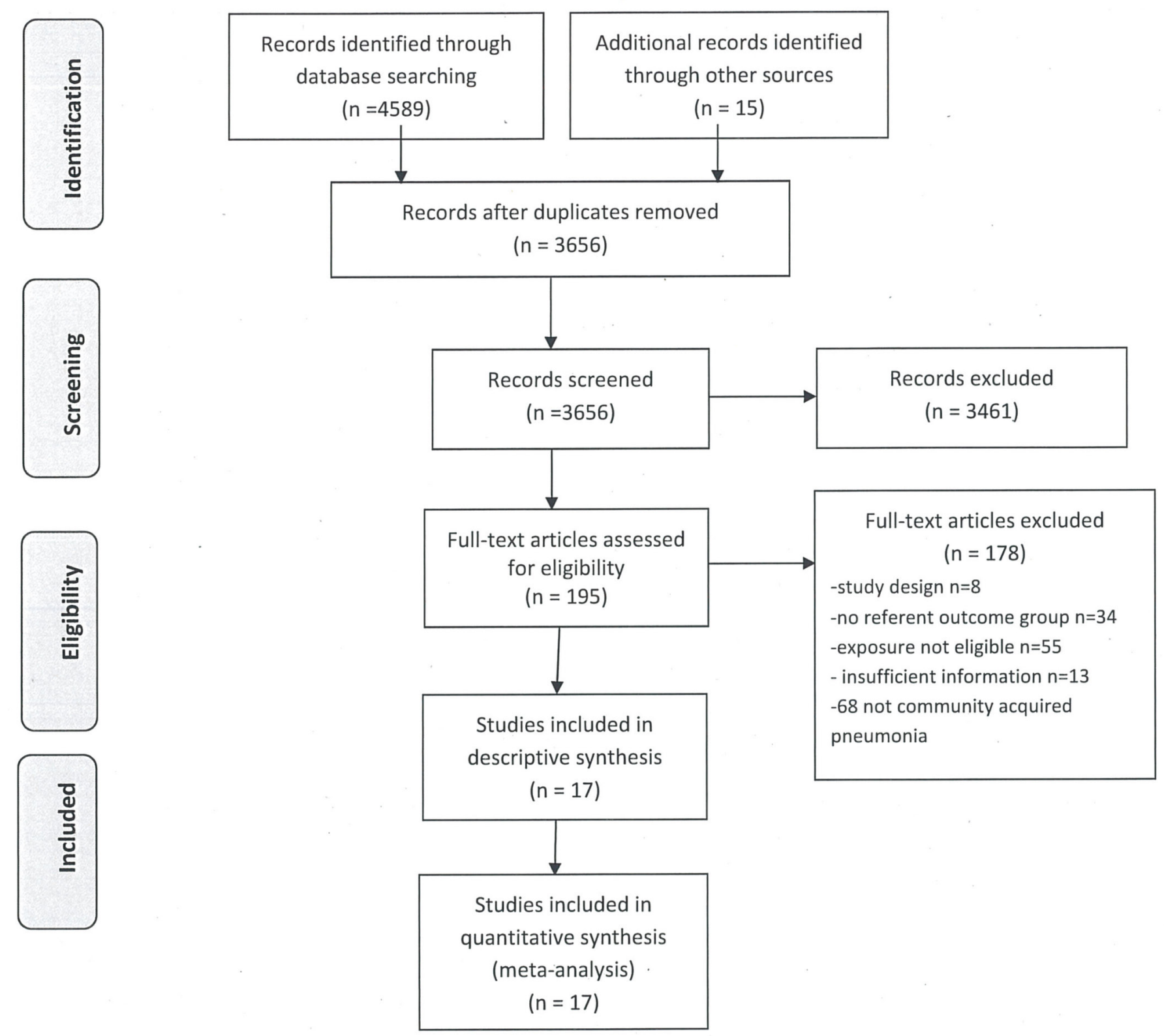

Figure 1 Study selection.

radiography. ${ }^{42-45} 48 \mathrm{~A}$ further seven studies ascertained CAP using ICD codes ${ }^{35} 3638404146$ and medical records, ${ }^{46}$ and two studies used self-report interviews. ${ }^{39} 49$ The remaining study ascertained CAP via physician diagnosis using medical records. ${ }^{10}$

The methodological quality of the case-control, cohort and cross-sectional studies ranged from 5 to 8 , with a median score of 6 . Ten studies were deemed to be of high quality (>6 score), ${ }^{10} 35$ 37-39 $414345-47$ whereas lower scores tended to arise from failure to adjust for confounders or using self-reported methods to ascertain alcohol consumption. The results of the quality assessment are presented in detail in table 2.

\section{Meta-analysis findings}

Fourteen of the 17 included studies provided data from which pooled RRs could be estimated, and a pooled analysis of these studies found the risk of CAP to be significantly increased in people who consumed alcohol at all or in higher amounts, relative to those who consumed no or lower amounts of alcohol, respectively (pooled $\mathrm{RR}=1.83,95 \%$ CI 1.30 to $2.57, \mathrm{I}^{2}=91 \%$; figure 2 ). There was no evidence of publication bias detected visually via a funnel plot (see online supplementary figure E1) and statistically via Egger's asymmetry test $(p=0.596)$.

Subgroup analyses exploring the reason for heterogeneity in the meta-analysis of these 14 studies are presented in online supplementary table E3). Heterogeneity was not explained by study design (case-control, longitudinal/cohort, cross-sectional; p for subgroup differences $=0.07$ ), methodological quality (high vs low; $p=0.09$ ) or gender (male vs female; $\mathrm{p}=0.74$ ). However, significant differences were found according to adjustment for 


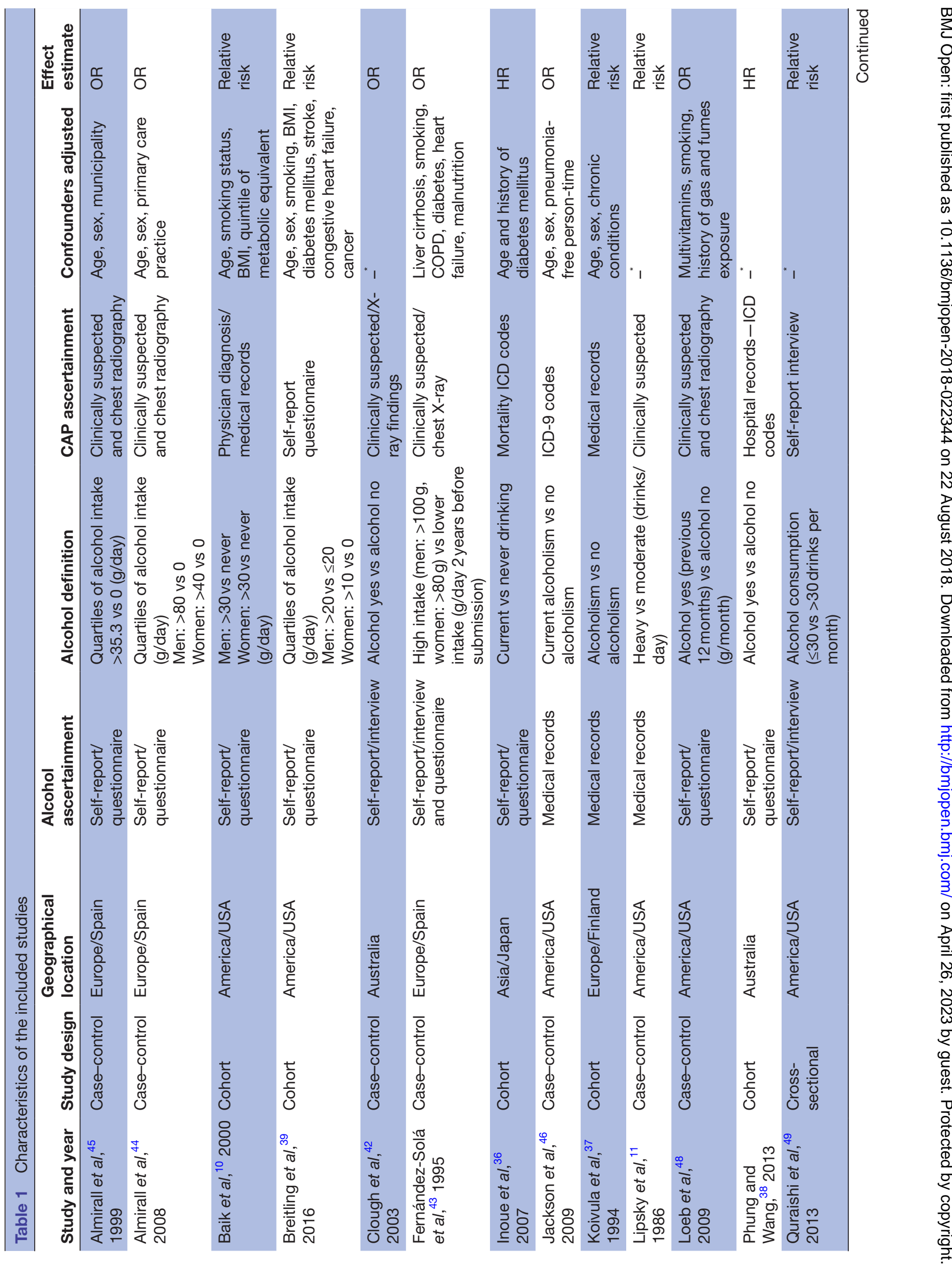




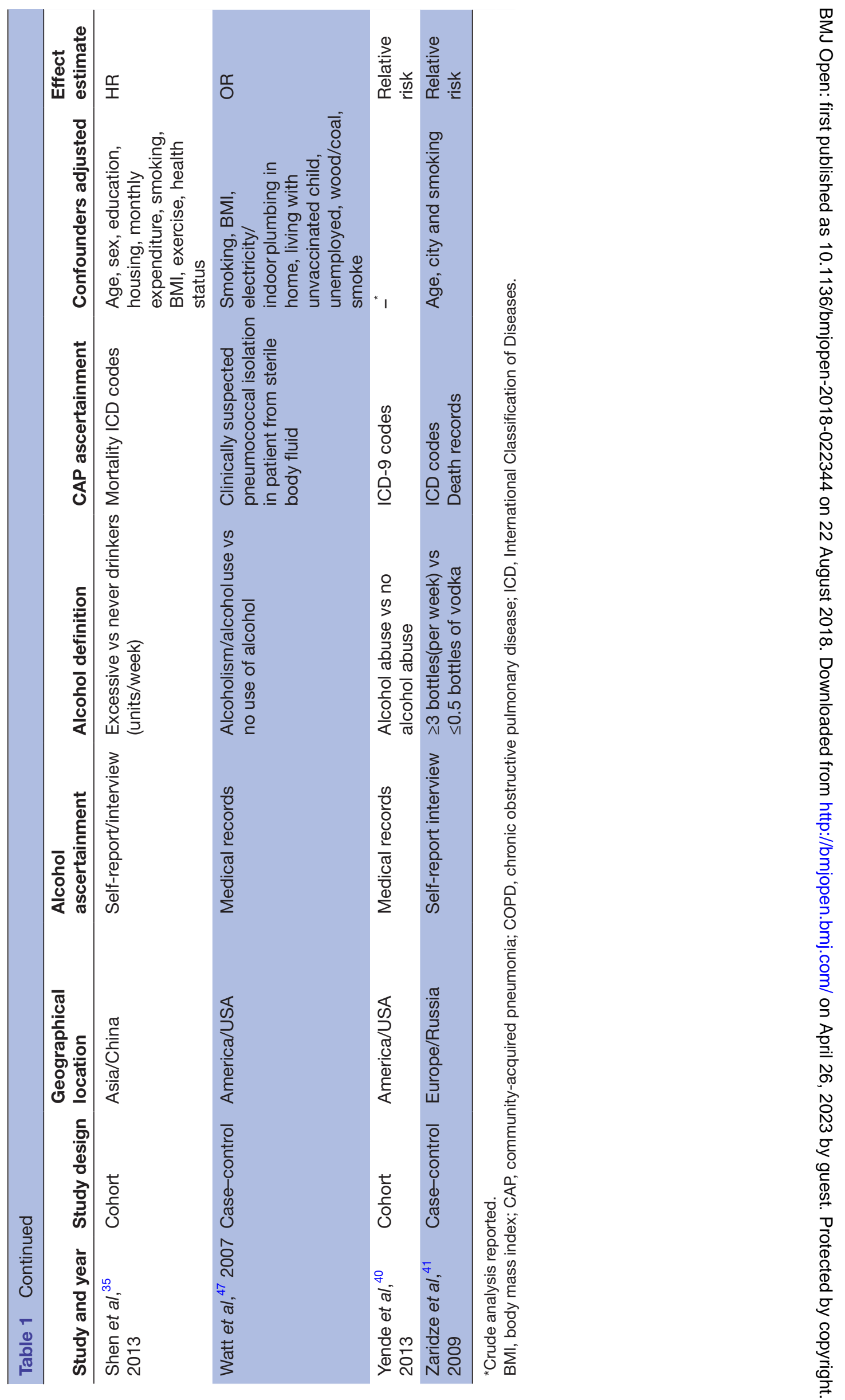


Table 2 Quality assessment: Newcastle-Ottawa Scale

\begin{tabular}{|c|c|c|c|c|}
\hline \multirow[b]{2}{*}{ Study, year } & \multicolumn{4}{|c|}{ Number of stars } \\
\hline & Selection* & Comparability & Exposure & Overall \\
\hline Almirall et al, ${ }^{45} 1999$ & 4 & 1 & 1 & $6 / 9$ \\
\hline Baik et $a l,{ }^{10} 2000$ & 4 & 2 & 2 & $8 / 9$ \\
\hline Breitling et al, ${ }^{39} 2016$ & 3 & 2 & 2 & $5 / 9$ \\
\hline Fernández-Solá et al, ${ }^{43} 1995$ & 3 & 2 & 1 & $6 / 9$ \\
\hline Inoue et $a l,{ }^{36} 2007$ & 3 & 1 & 1 & $5 / 9$ \\
\hline Jackson et al, ${ }^{46} 2009$ & 4 & 1 & 1 & $6 / 9$ \\
\hline Koivula et al, ${ }^{37} 1994$ & 4 & 1 & 3 & $8 / 9$ \\
\hline Lipsky et al, ${ }^{11} 1986$ & 3 & 0 & 2 & $5 / 9$ \\
\hline Shen et $a l,{ }^{35} 2013$ & 3 & 2 & 3 & $8 / 9$ \\
\hline Watt et al, ${ }^{47} 2007$ & 4 & 2 & 1 & $7 / 9$ \\
\hline Yende et $a l,{ }^{40} 2013$ & 4 & 0 & 2 & $6 / 9$ \\
\hline Zaridze et al, 2009 & 3 & 2 & 1 & $6 / 9$ \\
\hline
\end{tabular}

*Maximum 4 stars.

†Maximum 2 stars.

$\ddagger$ Maximum 3 stars.

confounders (adjusted vs unadjusted; $\mathrm{p}=0.03$ ), continent of study (America, Europe, Australia; $p=0.0003$ ), and ascertainment of CAP (clinical diagnosis vs death records; $\mathrm{p}=0.002$ ). Furthermore no difference was found for studies that presented OR estimates compared with studies that presented RR estimates ( $p$ for subgroup differences $=1.00$ ).

Additionally, no significant differences were found by the definition of the reference group for alcohol consumption ( $\mathrm{p}=0.39$; figure 2$)$. However, high heterogeneity $\left(\mathrm{I}^{2}=95 \%\right)$ was detected within the second subgrouping which used the lowest category of exposure as the reference group, where the following definitions were used: no alcoholism, ${ }^{3746}$ no alcohol abuse, ${ }^{40}$ moderate drinking, ${ }^{11}$ $\leq 30$ drinks $/$ month,${ }^{49} \leq 0.5$ bottles of vodka, ${ }^{41}<100 \mathrm{~g} /$ day for men and $<80 \mathrm{~g}$ /day for women, ${ }^{43}$ and $<20 \mathrm{~g} /$ day and $<10 \mathrm{~g} /$ day for men and women, respectively ${ }^{39}$; however, the gradient of exposure did not seem to be related to the magnitude of effect.

A sensitivity analysis restricted to the six studies which provided smoking-adjusted estimates found a larger magnitude of effect compared with the main analysis (pooled RR=2.01, 95\% CI 1.25 to 3.23, $\mathrm{I}^{2}=93 \%, 6$ studies). Similarly the studies that provided age-adjusted effect estimates found a risk of 1.90 (pooled $\mathrm{RR}=1.90,95 \% \mathrm{CI}$ 1.20 to $3.02, \mathrm{I}^{2}=93 \%, 7$ studies).

The remaining three studies presented effect estimates as HRs, ${ }^{35} 3638$ and a pooled analysis of these studies estimated an HR for CAP in relation to alcohol consumption of 0.90 (pooled $\mathrm{HR}=0.90,95 \%$ CI 0.79 to $1.03, \mathrm{I}^{2}=0,3$ studies).

Two studies assessing the effect of alcohol on pneumococcal disease-specific strains of pneumonia were identified. ${ }^{1147}$ A pooled analysis of these studies found that there was more than a doubling of risk of $S$. pneumoniae CAP in people who consumed alcohol $(\mathrm{RR}=2.16,95 \% \mathrm{CI}$ 1.05 to $4.48, \mathrm{I}^{2}=42 \%$ ).

\section{Biological gradient meta-analysis}

Five of the included studies provided data enabling a dose-response meta-analysis, ${ }^{1041424445}$ of which one used a cohort design (data reported separately for men and women) and four were case-control studies. A pooled analysis of the dose-response data from the cohort study found no significant gradient in the quantity of alcohol associated with the risk of CAP ( $p$ for trend=0.136). In contrast, the pooled analysis of the dose-response data from the four case-control studies indicated that there was a significant gradient in the quantity of alcohol associated with an $8 \%$ increase in the risk of CAP for every 10-20 g of pure alcohol consumed per day (equivalent to 1 drink/day) (pooled $\mathrm{RR}=1.08,95 \%$ CI 1.06 to 1.09 , $\mathrm{p}<0.0001$; figure 3 ).

\section{DISCUSSION}

Alcohol consumption is a recognised and avoidable risk factor for a range of diseases and injuries, including 




Figure 2 Forest plot of alcohol consumption and risk of community-acquired pneumonia (CAP): subgroup analysis based on reference group (never drinking vs lowest drinking category).

neuropsychiatric conditions, gastrointestinal and cardiovascular diseases, cancer, suicide, violence and tuberculosis. ${ }^{50}$ To date, however, the association between alcohol consumption and pneumonia risk has attracted relatively little attention.

\section{Summary of the findings}

This meta-analysis of studies published over the past 30 years demonstrates a clear and statistically significant

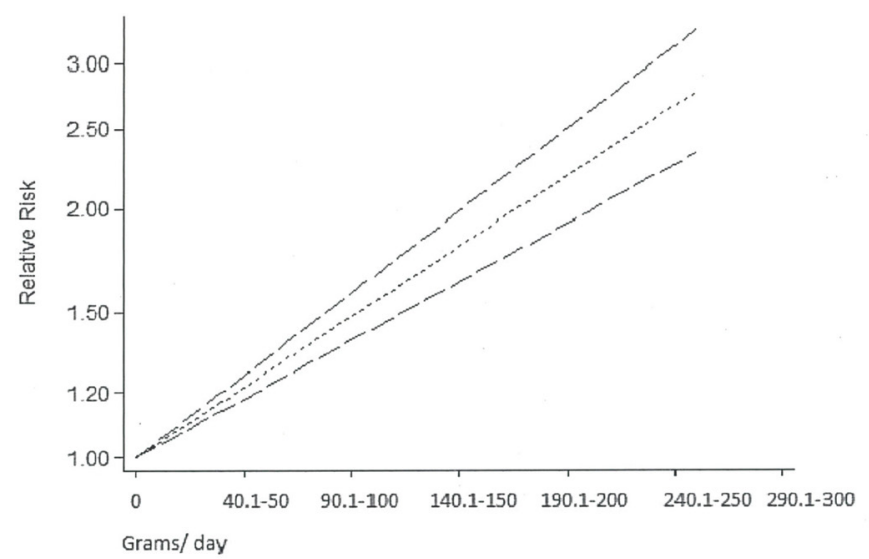

Figure 3 Linear dose-response meta-analysis for the association between alcohol intake categories (g/day) and the risk of community-acquired pneumonia. relation between alcohol consumption and the risk of CAP. The effect was strong, with a 1.8-fold increase in risk among those who consumed alcohol at all or in higher amounts, relative to those who consumed no or lower amounts of alcohol, respectively, and significantly related to the level of intake, with no evidence of publication bias. The dose-response analysis indicated that consuming drinks that contain 10-20 g of alcohol per day was linked to an $8 \%$ increased risk of acquiring CAP. Furthermore, the findings of the subgroup analysis indicated significant differences in the risk of pneumonia according to continent of the study, with Europe having the highest rate (threefold) for CAP risk.

\section{Strengths and limitations}

This study represents a comprehensive review of the global literature with no language restriction, making this analysis the most complete to date and our findings likely to be generalisable. There was significant heterogeneity between the studies in our analysis, but our subgroup analyses indicate that this arose primarily from the continent in which the study was carried out (America, Europe, Australia), adjustment for confounders and the ascertainment of CAP (death vs clinical diagnosis). Misclassification bias arising from inclusion of non-drinkers in the lowest category of alcohol intake in some studies can be 
another possible limitation in our review, but will result in a more conservative estimate of effect. A dose-response relationship was identified. However the included studies did not report dose-response relations separately for men and women, so we are unable to carry out a comparative analysis. Furthermore, confounding as a result of the existence of other factors that were not usually adjusted for in the included studies (eg, socioeconomic status, malnutrition) could not be explored.

\section{Comparison with other studies}

Our findings extend those of an earlier review and meta-analysis carried out in 2010. ${ }^{19}$ Another review focused on the risk factors for invasive pneumococcal diseases indicated an elevated risk for invasive pneumococcal disease due to alcohol consumption in six of the four studies included in the meta-analysis model ${ }^{51}$ Likewise, another recent meta-analysis indicated an elevated risk for invasive pneumococcal disease due to alcohol consumption in six of the four studies included in the meta-analysis model. ${ }^{52}$ Similarly our separate meta-analysis focused on pneumococcal infections including two of these studies, due to our eligibility criteria, showed an elevated risk for pneumococcal acquisition.

A previous systematic review and meta-analysis found that people with a daily alcohol consumption of either 24 $\mathrm{g}, 60 \mathrm{~g}$ and $120 \mathrm{~g}$ have a $12 \%, 33 \%$ and $76 \%$ increased risk of CAP, respectively. ${ }^{19}$ Our dose-response analysis generated a slightly less strong effect, of an $8 \%$ increase in risk per 10-20 $\mathrm{g}$ of (pure) alcohol consumed per day.

A general systematic review published by Almirall et al in $2017^{53}$ focused on the risk factors of CAP, but provided only a narrative summary of findings and stating that no definite conclusion could be drawn. In contrast, our review found evidence of a doubling in the risk of CAP in people who consumed alcohol. Furthermore, our demonstration of a significant exposure-response association increases the likelihood, given the strength of the observed association and its consistency across a range of subgroups, that the observed association is causal. Further evidence of causality arises from studies demonstrating that alcohol consumption impairs alveolar macrophages and increases carriage of pneumonia pathogens. ${ }^{151654}$

\section{Clinical implications}

The findings from the present review highlight the need to address high alcohol consumption as a means to prevent CAP. Clinicians managing patients with pneumonia could, for example, counsel reducing alcohol intake as a means to prevent further episodes, and those addressing high alcohol consumption in more general terms could add an increased risk of pneumonia as a further reason to reduce intake.

Our findings also have implications for public health: in Europe, for example, the estimated annual cost of CAP is approximately $€ 10.1$ billion $^{55}$ and might be reduced substantially by more proactive clinical and public health measures to reduce alcohol consumption.

\section{CONCLUSION}

Our findings thus provide clear evidence that alcohol increases the risk of pneumonia. Informing people who drink alcohol of this risk, especially those who consume high levels of alcohol, both in clinical contacts and through public health policy, may therefore help to prevent this disease.

Acknowledgements The authors thank Erica Brasil, Magdalena Opazo-Breton and Yue Huang from the University of Nottingham for their help in translations.

Contributors ES, JB and JL-B designed the study and wrote the protocol. ES wrote the search strategy and undertook the literature searches, and wrote the draft of the manuscript. ES and JL-B undertook study screening, data extraction and quality assessment. ES undertook all data analyses, supervised by JL-B. All authors contributed to the interpretation of the findings. JB and JL-B provided critical revisions to the article, and all authors approved the final version of the article to be published. ES acts as guarantor of the manuscript.

Funding This work was supported by the Medical Research Council (grant number MR/K023195/1). The UK Centre for Tobacco and Alcohol Studies (http://www. ukctas.net), and the British Heart Foundation, Cancer Research UK, the Economic and Social Research Council, and the National Institute for Health Research, under the auspices of the UK Clinical Research Collaboration, are gratefully acknowledged.

Competing interests None declared.

Patient consent Not required.

Ethics approval Ethics approval was not required for this work.

Provenance and peer review Not commissioned; externally peer reviewed.

Data sharing statement No data sharing available.

Open access This is an open access article distributed in accordance with the Creative Commons Attribution 4.0 Unported (CC BY 4.0) license, which permits others to copy, redistribute, remix, transform and build upon this work for any purpose, provided the original work is properly cited, a link to the licence is given, and indication of whether changes were made. See: https://creativecommons.org/ licenses/by/4.0/.

\section{REFERENCES}

1. Murphy SL, Kochanek KD, Xu J, et al. Mortality in the United States, 2014. NCHS data brief 2015;229:1-8.

2. World Health Organization. Pneumonia Fact Sheet;World Health Organization Report. Geneva, Switzerland: WHO, 2016.

3. Rozenbaum MH, Pechlivanoglou P, van der Werf TS, et al. The role of Streptococcus pneumoniae in community-acquired pneumonia among adults in Europe: a meta-analysis. Eur J Clin Microbiol Infect Dis 2013;32:305-16.

4. Ruuskanen O, Lahti E, Jennings LC, et al. Viral pneumonia. The Lancet 2011;377:1264-75.

5. Woodhead M. Community-acquired pneumonia in Europe: causative pathogens and resistance patterns. Eur Respir J Suppl 2002;36-20s-7.

6. Jain S, Self WH, Wunderink RG, et al. Community-Acquired Pneumonia Requiring Hospitalization among U.S. Adults. N Engl J Med 2015;373:415-27.

7. Walden AP, Clarke GM, McKechnie S, et al. Patients with community acquired pneumonia admitted to European intensive care units: an epidemiological survey of the GenOSept cohort. Crit Care 2014;18:R58.

8. European Commission. Health statistics, Atlas on mortality in the European Union. Luxembourg: Office for Official Publications of the European Communities, 2008.

9. Jain S, Williams DJ, Arnold SR, et al. Community-acquired pneumonia requiring hospitalization among U.S. children. N Engl J Med 2015;372:835-45.

10. Baik I, Curhan GC, Rimm EB, et al. A prospective study of age and lifestyle factors in relation to community-acquired pneumonia in US men and women. Arch Intern Med 2000;160:3082-8.

11. Lipsky BA, Boyko EJ, Inui TS, et al. Risk factors for acquiring pneumococcal infections. Arch Intern Med 1986;146:2179-85. 
12. Kohlhammer $\mathrm{Y}$, Schwartz $\mathrm{M}$, Raspe $\mathrm{H}$, et al. [Risk factors for community acquired pneumonia (CAP). A systematic review]. Dtsch Med Wochenschr 2005;130:381-6.

13. Simet SM, Sisson JH. Alcohol's Effects on Lung Health and Immunity. Alcohol Res 2015;37:199.

14. Vinogradova Y, Hippisley-Cox J, Coupland C. Identification of new risk factors for pneumonia: population-based case-control study. $\mathrm{Br}$ $J$ Gen Pract 2009;59:329-38.

15. Mehta AJ, Guidot DM. Pathophysiology Review Series: Alcohol Abuse, the Alveolar Macrophage, and Pneumonia. The American journal of the medical sciences 2012;343:244.

16. Kershaw CD, Guidot DM. Alcoholic lung disease. Alcohol Res Health 2008;31:66-76.

17. Moss M. Epidemiology of sepsis: race, sex, and chronic alcohol abuse. Clin Infect Dis 2005;41 Suppl 7-S490-S497.

18. Marsano L. Alcohol and malnutrition. Alcohol Research and Health 1993;17:284.

19. Samokhvalov AV, Irving HM, Rehm J. Alcohol consumption as a risk factor for pneumonia: a systematic review and meta-analysis. Epidemiol Infect 2010;138:1789-95.

20. Moher D, Liberati A, Tetzlaff J, et al. Preferred reporting items for systematic reviews and meta-analyses: the PRISMA statement. Ann Intern Med 2009;151:264-9.

21. Stroup DF, Berlin JA, Morton SC, et al. Meta-analysis of observational studies in epidemiology: a proposal for reporting. Journal of American Medical Association 2000;283:2008-12.

22. SIGN. Search Filters, Obseravtional studies. http://www.sign.ac.uk/ methodology/filters.html\#obs (accessed 4 Dec 2015).

23. Cochrane Library. http://www.cochranelibrary.com/ (accessed 4 Dec 2015).

24. Centers for Disease Control and Prevention (CDC). Alcohol \& Public Health, Fact Sheets - Alcohol Use and Your Health. http://www.cdc. gov/alcohol/fact-sheets/alcohol-use.htm (accessed 10 Dec 2015).

25. U.S Department of Health and Human Services and U.S Department of Agriculture. 2015-2020.Dietary Guidelines for Americans. 8th Edition. Washington: DC, 2015. https://health.gov/dietaryguidelines/ 2015/resources/2015-2020_Dietary_Guidelines.pdf. (accessed 15 Dec 2015).

26. Wells G, Shea B, O'connell D, et al. The Newcastle-Ottawa Scale (NOS) for assessing the quality of nonrandomised studies in metaanalyses. http://www.ohri.ca/programs/clinical_epidemiology/oxford. asp (accessed 10 Feb 2016).

27. Deeks J. Swots corner: what is an odds ratio. Bandolier 1996;3:6-7.

28. Green S, Higgins J. Cochrane handbook for systematic reviews of interventions. Version, 2005.

29. Higgins JP, Thompson SG. Quantifying heterogeneity in a metaanalysis. Stat Med 2002;21:1539-58.

30. Hill AB. The environment and disease: association or causation?: SAGE Publications, 1965

31. Greenland S, Longnecker MP. Methods for trend estimation from summarized dose-response data, with applications to meta-analysis. Am J Epidemiol 1992;135:1301-9.

32. Orsini N, Bellocco R, Greenland S. Generalized least squares for trend estimation of summarized dose-response data. Stata Journal 2006;6:40.

33. Berlin JA, Longnecker MP, Greenland S. Meta-analysis of epidemiologic dose-response data. Epidemiology 1993;4:218-28.

34. Orsini N. From floated to conventional confidence intervals for the relative risks based on published dose-response data. Comput Methods Programs Biomed 2010;98:90-3.

35. Shen C, Ni MY, Schooling CM, et al. Alcohol use and death from respiratory disease in a prospective Chinese elderly cohort study in Hong Kong. Prev Med 2013;57:819-23.
36. Inoue $\mathrm{Y}$, Koizumi A, Wada $\mathrm{Y}$, et al. Risk and protective factors related to mortality from pneumonia among middleaged and elderly community residents: the JACC Study. J Epidemiol 2007:17:194-202.

37. Koivula I, Sten M, Mäkelä PH. Risk factors for pneumonia in the elderly. Am J Med 1994;96:313-20.

38. Phung DT, Wang Z. Risk of pneumonia in relation to body mass index in Australian Aboriginal people. Epidemiol Infect 2013;141:2497-502.

39. Breitling LP, Saum K-U, Schöttker B, et al. Pneumonia in the Noninstitutionalized Older Population: A prospective observational study over 11 years. Deutsches Ärzteblatt International 2016;113:607.

40. Yende S, Alvarez K, Loehr L, et al. Epidemiology and long-term clinical and biologic risk factors for pneumonia in communitydwelling older Americans: analysis of three cohorts. Chest 2013;144:1008-17.

41. Zaridze D, Brennan P, Boreham J, et al. Alcohol and cause-specific mortality in Russia: a retrospective case-control study of 48,557 adult deaths. Lancet 2009;373:2201-14.

42. Clough AR, Wang Z, Bailie RS, et al. Case-control study of the association between kava use and pneumonia in eastern Arnhem and Aboriginal communities (Northern Territory, Australia). Epidemiol Infect 2003;131:627-35.

43. Fernández-Solá J, Junqué A, Estruch R, et al. High alcohol intake as a risk and prognostic factor for community-acquired pneumonia. Arch Intern Med 1995;155:1649-54.

44. Almirall J, Bolíbar I, Serra-Prat M, et al. New evidence of risk factors for community-acquired pneumonia: a population-based study. Eur Respir J 2008;31:1274-84

45. Almirall J, Bolíbar I, Balanzó X, et al. Risk factors for communityacquired pneumonia in adults: a population-based case-control study. Eur Respir J 1999;13:349-55.

46. Jackson ML, Nelson JC, Jackson LA. Risk factors for communityacquired pneumonia in immunocompetent seniors. J Am Geriatr Soc 2009;57:882-8.

47. Watt JP, O'Brien KL, Benin AL, et al. Risk factors for invasive pneumococcal disease among Navajo adults. Am J Epidemiol 2007:166:1080-7.

48. Loeb M, Neupane B, Walter SD, et al. Environmental risk factors for community-acquired pneumonia hospitalization in older adults. J Am Geriatr Soc 2009;57:1036-40.

49. Quraishi SA, Bittner EA, Christopher KB, et al. Vitamin D status and community-acquired pneumonia: results from the third National Health and Nutrition Examination Survey. PLoS One 2013;8:e81120.

50. World Health Organization. Global status report on alcohol and health, 2014.

51. Boé DM, Vandivier RW, Burnham EL, Moss M, et al. Alcohol abuse and pulmonary disease. J Leukoc Biol 2009;86:1097-104.

52. Cruickshank HC, Jefferies JM, Clarke SC. Lifestyle risk factors for invasive pneumococcal disease: a systematic review. BMJ Open 2014; 4:e005224.

53. Almirall J, Serra-Prat M, Bolíbar I, et al. Risk Factors for Community-Acquired Pneumonia in Adults: A Systematic Review of Observational Studies. Respiration 2017:94:299-311.

54. Dao TT, Liebenthal D, Tran TK, et al. Klebsiella pneumoniae oropharyngeal carriage in rural and urban Vietnam and the effect of alcohol consumption. PLoS One 2014;9:e91999.

55. Welte T, Torres A, Nathwani D. Clinical and economic burden of community-acquired pneumonia among adults in Europe. Thorax 2012;67:71-9. 